\title{
GUide sheath Advancement and aspiRation in the Distal petrocavernous internal carotid artery (GUARD) Technique during Thrombectomy Improves Reperfusion and Clinical Outcomes
}

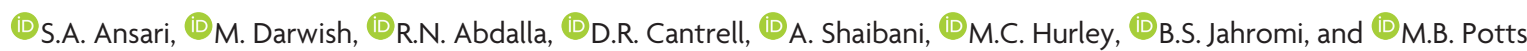

\begin{abstract}
BACKGROUND AND PURPOSE: Adjunctive techniques to stent retriever thrombectomy include balloon-guide catheters and/or distal access catheters for aspiration. We describe a novel technique using a flexible, 6 French 088 distal guide sheath advanced past the skull base to augment mechanical thrombectomy. We studied the relative safety and efficacy of this technique in the setting of a combined stent retriever-distal access catheter aspiration thrombectomy protocol.
\end{abstract}

MATERIALS AND METHODS: We performed a retrospective case-control study of intracranial internal carotid artery or M1-M2 middle cerebral artery occlusions requiring mechanical thrombectomy. Patients were divided into 2 groups based on thrombectomy techniques: conventional stent retriever with distal access catheter aspiration without (standard) and with adjunctive GUide sheath Advancement and aspiRation in the Distal petrocavernous internal carotid artery (GUARD). Using propensity score matching, we compared procedural safety, reperfusion efficacy using the modified Thrombolysis in Cerebral Infarction scale and clinical outcomes with the modified Rankin Scale.

RESULTS: In comparing the GUARD (45 patients) versus standard (45 matched case controls) groups, there were no significant differences in demographics, NIHSS presentations, IV rtPA use, median onset-to-groin puncture times, procedural complications, symptomatic intracranial hemorrhage, or mortality. The GUARD group demonstrated significantly higher successful mTICI $\geq 2 \mathrm{~b}$ reperfusion rates ( $98 \%$ versus $80 \%, P=.015$ ) and improved functional $m R S \leq 2$ outcomes ( $67 \%$ versus $43 \%, P=.04$ ), with independent effects of the GUARD technique confirmed in a multivariable logistic regression model.

CONCLUSIONS: The GUARD technique during mechanical thrombectomy with combined stent retrieval-distal access catheter aspiration is safe and effective in improving reperfusion and clinical outcomes.

ABBREVIATIONS: $B G C=$ balloon-guide catheter; $\mathrm{DAC}=$ distal access catheter; $\mathrm{DGS}=$ distal guide sheath; GUARD = GUide sheath Advancement and aspiRation in the Distal petrocavernous internal carotid artery; $\mathrm{F}=$ French; $\mathrm{mTICl}=$ modified Thrombolysis in Cerebral Infarction; $\mathrm{mRS}=$ modified Rankin Scale; NIHSS = National Institutes of Health Stroke Scale; ASPECTS = Alberta Stroke Program Early CT Score; ICA = internal carotid artery; MCA = middle cerebral artery; IV rtPA = intravenous recombinant tissue plasminogen activator; $\mathrm{SICH}=$ symptomatic intracranial hemorrhage; ELVO = emergent large-vessel occlusions

E ndovascular mechanical thrombectomy for emergent largevessel occlusions (ELVO) is the standard of care in acute ischemic stroke treatment as confirmed by multiple randomized controlled trials. ${ }^{1,2}$ The rate of successful recanalization and effective reperfusion (TICI $\geq 2 \mathrm{~b}$ ) was attributed to modern stent-retriever technology and was estimated to be $71 \%$ in the pooled Highly Effective Reperfusion evaluated in Multiple Endovascular Stroke

Received March 26, 2019; accepted after revision June 17

From the Departments of Radiology (S.A.A., M.D., R.N.A., D.R.C., A.S., M.C.H., B.S.J., M.B.P.), Neurology (S.A.A.), and Neurological Surgery (S.A.A., D.R.C., A.S., M.C.H., B.S.J., M.B.P.), Feinberg School of Medicine, Northwestern University, Chicago, Illinois; Department of Neurology (M.D.), Assiut University, Assiut, Egypt; and Department of Radiology (R.N.A.), Ain Shams University, Cairo, Egypt.

Please address correspondence to Sameer A. Ansari, MD, PhD, Departments of Radiology, Neurology, and Neurological Surgery, Northwestern University,

Feinberg School of Medicine, 676 N St. Clair St, Suite 800, Chicago, IL 60611-2927;

e-mail: s-ansari@northwestern.edu

http://dx.doi.org/10.3174/ajnr.A6132
Trials (HERMES) ${ }^{2}$ meta-analysis, corresponding to better functional outcomes for treatment-versus-control groups (46\% versus $26.5 \%$, mRS $\leq 2$ ), respectively. Subsequently, several thrombectomy devices and protocols were developed to optimize angiographic and clinical outcomes.

Early adaptations in thrombectomy techniques can be classified into 3 groups: traditional stent retriever thrombectomy, a distal access catheter (DAC) aspiration technique (A Direct Aspiration First Pass Technique [ADAPT] or Forced Aspiration Suction Thrombectomy [FAST]), ${ }^{3,4}$ and a combined stent retriever with DAC aspiration approach (SOLUMBRA, Continuous Aspiration Prior To Intracranial Vascular Embolectomy [CAPTIVE], or Stent retriever Assisted Vacuum-locked Extraction [SAVE] $.^{5-7} \mathrm{Al}-$ though the Contact Aspiration vs Stent Retriever for Successful Revascularization (ASTER) and Aspiration thrombectomy versus stent retriever thrombectomy as first-line approach for large ves- 
sel occlusion (COMPASS) randomized controlled trials demonstrated equivalency of stent retriever versus contact aspiration thrombectomy, these trials did not study combined techniques or control for adjunctive aspiration through a proximal guide-catheter/sheath or balloon guide catheter (BGC). ${ }^{8,9}$ Compared with stent retriever thrombectomy alone, the combination of stent retrievers with DAC aspiration reported higher rates of successful (>80\%) and first-pass (37\%-72\%) reperfusion, indicating lower rates of thrombus fragmentation. ${ }^{5-7}$

BGCs have also been extensively employed to minimize the risk of thrombus fragmentation and embolization via flow modification or flow arrest in the proximal parent artery. A metaanalysis by Brinjikji et al ${ }^{10}$ showed BGC utilization to be more effective in successful TICI $2 \mathrm{~b} / 3$ reperfusion (78.9\% versus $67 \%$ ), single-pass recanalization $(63.1 \%$ versus $45.2 \%)$, reduced procedure times (70.5 versus 90.9 minutes), and improved functional outcomes (59.7\% versus $43.8 \%$, mRS 0-2). ${ }^{11}$ Further complementary techniques were described by Massari et al ${ }^{12}$ (Aspiration Retriever Technique for Stroke [ARTS]) and Stampfl et $\mathrm{al}^{13}$ that combined stent retriever thrombectomy with both BGC and DAC aspiration. Maegerlein et $\mathrm{al}^{14}$ directly compared this type of PRoximal balloon Occlusion TogEther with direCt Thrombus aspiration during stent retriever thrombectomy (PROTECT) technique against stent retriever thrombectomy with $\mathrm{DAC}$ aspiration alone; the PROTECT technique achieved higher rates of successful TICI $\geq 2 \mathrm{~b}$ ( $100 \%$ versus $78 \%$ ) and complete TICI $3(70 \%$ versus $39 \%$ ) reperfusion, as well as shorter procedure times (29 versus 40 minutes).

We investigated a novel method of flow modification via coaxial advancement and aspiration of a flexible 6F 088 distal guide sheath (DGS) past the skull base during mechanical thrombectomy, analogous to BGC and DAC adjunctive techniques. We hypothesized that this GUide sheath Advancement and aspiRation in the Distal petrocavernous internal carotid artery (GUARD) technique could reduce antegrade flow as well as clot-retrieval distance, allowing more efficient and complete reperfusion. We examined the safety, technical and clinical efficacy of the adjunctive GUARD technique compared with our standard combined stent retriever with DAC aspiration thrombectomy protocol in a propensity score-matched patient cohort.

\section{MATERIALS AND METHODS}

Institutional review board approval was obtained for a retrospective case-control study of all patients with acute ischemic stroke secondary to anterior circulation ELVO who underwent mechanical thrombectomy at 3 comprehensive stroke centers by 5 neurointerventionalists, using a standardized thrombectomy protocol from November 2016 to August 2018. Patient and imaging criteria for mechanical thrombectomy included severe acute ischemic stroke symptoms (NIHSS $\geq 5$ ), presentation $<24$ hours from last known well, CT ASPECTS $\geq 6$, CT angiography confirmed ELVO (intracranial ICA or M1-M2 MCA), and CT perfusion (CTP) profile consistent with the Endovascular Therapy Following Imaging Evaluation for Ischemic Stroke (DEFUSE 3) ${ }^{15}$ or Clinical Mismatch in the Triage of Wake-Up and Late Presenting Strokes Undergoing Neuro- intervention with Trevo (DAWN) ${ }^{16}$ trial inclusion criteria if presenting $>6$ hours from last known well.

Cases were separated by thrombectomy technique into a standard group versus a novel GUARD group with technical adaptation as detailed below. Use of the adjunctive GUARD technique was first introduced by a single neurointerventionalist and subsequently adopted by the remaining practitioners during the study period. After initial data analysis of both groups, a propensity score-matched analysis to control for confounding variables provided equivalent case controls in the standard group for comparison against the GUARD group.

\section{Interventions: Standard-versus-GUARD Thrombectomy Techniques}

All procedures were initiated via transfemoral puncture using $6 \mathrm{~F} \times 80-100 \mathrm{~cm}$ guide sheaths (Flexor Shuttle, Cook; Neuron MAX, Penumbra; or AXS Infinity, Stryker), which were initially placed in the cervical ICA. All procedures used a triaxial system with coaxial advancement of large-bore DACs (ACE 60, ACE 64, or ACE 68; Penumbra) and 021/027 microcatheters using 0.014-/ 0.016 -inch microwires to cross the thromboembolus. Stent retrievers (Trevo XP ProVue Retriever, Stryker; Solitaire/Solitaire 2, Medtronic) were deployed with $>50 \%$ of the stent construct distal to the clot followed by immediate DAC advancement under continuous vacuum aspiration (MAX/Engine Pump, Penumbra) to the proximal aspect of the clot until cessation of flow indicated engagement of the thromboembolus and stent retriever. Next, the internal 021/027 stent delivery microcatheter was removed to maximize the DAC aspiration force. In the standard thrombectomy protocol, after $>5$ minutes to optimize clot integration, the combined stent retriever-DAC aspiration complex was removed from the cervical guide sheath under manual ( $>30 \mathrm{~mL})$ syringe aspiration.

In the modified GUARD technique, only flexible $6 \mathrm{~F} 088 \times$ 90-100 cm guide sheaths (Neuron MAX, Penumbra; or AXS Infinity, Stryker) were used. Following stent retriever deployment and DAC advancement under vacuum aspiration to engage the proximal aspect of the thromboembolus, the flexible 6F 088 DGS was further triaxially advanced across the skull base (horizontal petrous to posterior genu cavernous segment of the ICA) over the large-bore (6F) DAC, deployed stent retriever, and 021/027 stentdelivery microcatheter. After removal of the internal 021/027 stent-delivery microcatheter to maximize the DAC aspiration force and $\sim 5$ minutes to optimize clot integration with the stent retriever, the combined stent retriever-DAC aspiration complex was removed from the flexible 6F 088 DGS within the petrocavernous ICA, while continuous aspiration was applied to both the DAC (vacuum) and DGS (manual). Finally, the 6F 088 DGS was continuously aspirated and retracted into the cervical or proximal petrous segments of the ICA until blood return confirmed guidesheath clearance and no residual flow limitation (Fig 1).

\section{Data and Outcome Analysis}

All patient and imaging data were extracted from a prospectively maintained stroke intervention data base integrated across all comprehensive stroke centers, the electronic medical record, and/or PACS. We analyzed patient demographics (age, sex), presentations (NIHSS, IV rtPA), imaging selection (CT 


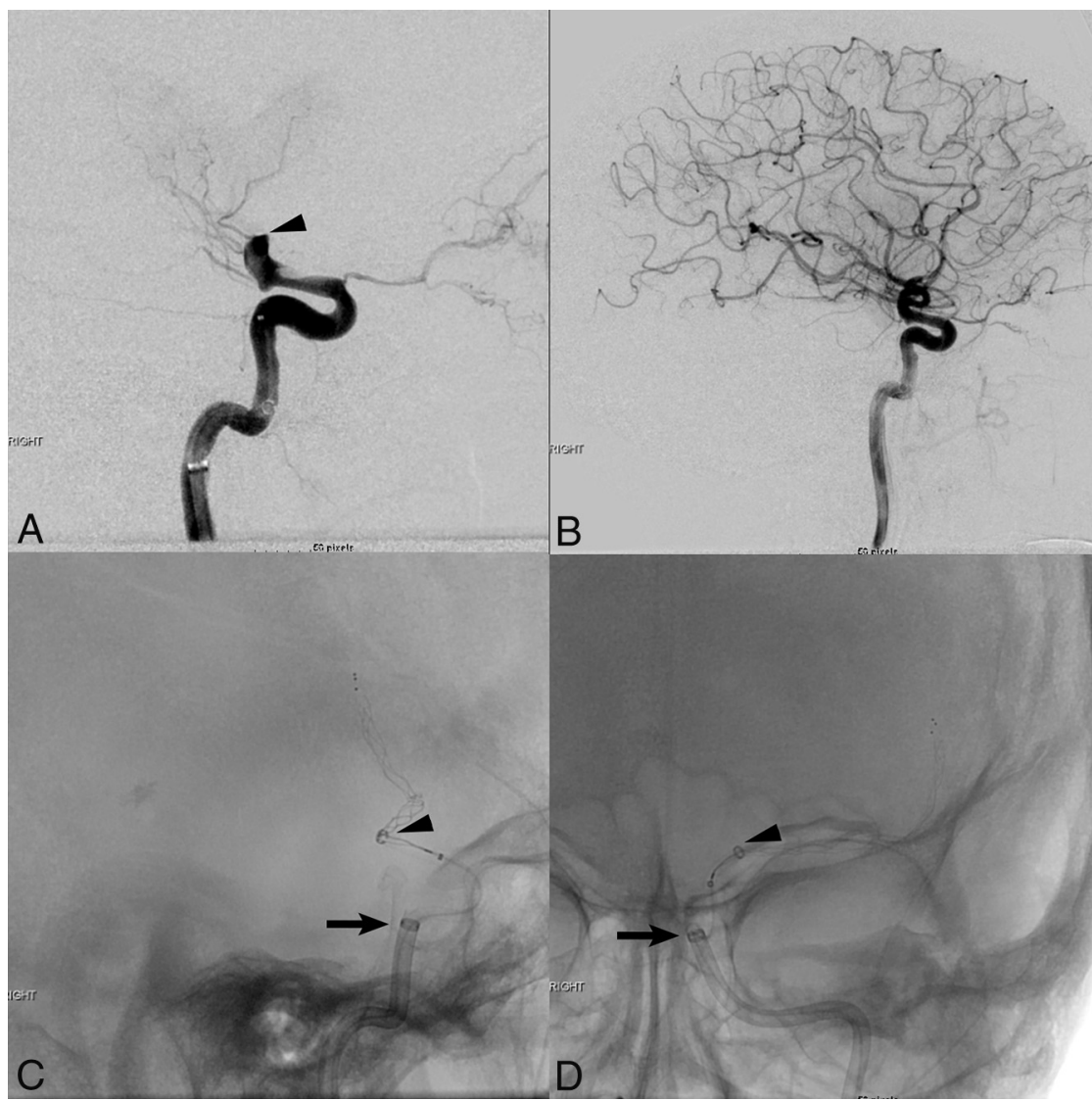

FIG 1. Lateral pre- $(A)$ and post- $(B)$ thrombectomy DSA images demonstrate complete TICI 3 reperfusion of a left ICA terminus (arrowhead) occlusion using the GUARD technique with distal guide sheath placement and combined stent retriever-distal access catheter aspiration thrombectomy. Fluoroscopic intraoperative images in the lateral $(C)$ and antero-posterior $(D)$ projections show stent retriever deployment across the supraclinoid ICA/MI MCA occlusion with advancement of a large-bore ACE 68 distal access catheter (arrowheads) to the proximal aspect of the clot under vacuum aspiration, and triaxial advancement of the Neuron Max 088 guide sheath (arrows) distally into the posterior genu cavernous segment of the left ICA.

ASPECTS/CTP), vessel occlusion locations, times to treatment (symptom onset to groin puncture or successful reperfusion), procedure times, angiographic outcomes (thrombectomy passes, mTICI scores), major (neurovascular) and minor (access site) procedural complications, SICH per the European Cooperative Acute Stroke Study (ECASS-3) criteria, ${ }^{17}$ clinical outcomes (mRS), and mortality. We adjudicated 2 study outcomes: angiographic and clinical outcomes. Angiographic outcomes were determined by measuring the mTICI score on post-thrombectomy cerebral angiography; each score reported during the procedure was graded and confirmed by a blinded neurointerventionalist who did not perform the procedure. Successful revascularization or reperfusion efficacy was defined as $\mathrm{mTICI} \geq 2 \mathrm{~b}$, and complete reperfusion was defined as mTICI $2 \mathrm{c} / 3 .{ }^{18}$ Clinical success was defined as a good functional neurological outcome measured by $\mathrm{mRS} \leq 2$, requiring concordance of separate assessments by both stroke neurology and neurointerventional surgery practitioners at 90-day follow-up. In cases of disagreement, the lower TICI and higher mRS scores were used for analysis of angiographic and clinical outcomes, respectively. Safety was assessed by comparing major procedural complications, $\mathrm{SICH}$, and mortality at 90 days in the GUARD relative to standard control groups.

\section{Statistical Analysis}

A propensity score-matched analysis was performed to compare patients who underwent thrombectomy treated by the GUARD-versus-standard techniques with a ratio of $1: 1$ using confounding variables on the initial data analysis: age, IV rtPA use, CTP selection, and previous stroke/TIA as predictors with a greedy Euclidian matching algorithm and matching tolerance of 0.2 (XLSTAT-Premium 2018.6; Addinsoft, Long Island City, New York). Baseline characteristics along with procedural and clinical outcomes were compared between the GUARD and standard groups using Mann-Whitney and Fisher exact tests for continuous and categorical variables, respectively, with statistical significance set at a $P$ value $<.05$ (SPSS 24; IBM, Armonk, New York).

A multivariable logistic regression analysis was performed to assess the independent effects of the GUARD technique by adjusting for outcome confounders. Prespecified dependent variables were set to either a good functional outcome ( $\mathrm{mRS} \leq 2$ ), successful or complete reperfusion; the independent variables included age, onset to groin puncture, IV rtPA use, occlusion location, and CTP selection. Results of the logistic regression analysis were reported as an adjusted odds ratio (aOR) with $95 \%$ confidence intervals (CI). All data were reported as percentages or median (range), except for the number of thrombectomy passes reported as a mean \pm standard deviation $(\mathrm{SD})$.

\section{RESULTS}

We identified 112 consecutive patients with acute ischemic stroke with anterior circulation ELVO who underwent mechanical thrombectomy with a standard stent retriever-DAC aspiration protocol during the study period, including 45 patients with an adjunctive DGS in the petrocavernous ICA composing the GUARD group. After propensity score 1:1 ratio matching, we identified 45/67 patients in the standard group (clot retrieval into a cervical ICA guide sheath) as case controls for direct comparison.

There were no significant differences in baseline age, sex, stroke risk factors (atrial fibrillation, hypertension, hyperlipidemia, diabetes mellitus, smoking, or prior TIA/stroke), initial NIHSS presentation, CT ASPECTS and/or CTP imaging selection, IV rtPA use, vessel occlusion location (including tandem ICA/MCA occlusions), or symptom onset-to-groin puncture time between the GUARD and standard groups (Table 1). Although there remained a trend toward younger patients being 
Table 1: Comparison of baseline demographics, presentations, procedural efficacy, and safety between the GUARD and standard thrombectomy techniques

\begin{tabular}{|c|c|c|c|}
\hline & $\begin{array}{l}\text { GUARD } \\
(n=45)\end{array}$ & $\begin{array}{l}\text { Standard } \\
(n=45)\end{array}$ & $\begin{array}{c}P \\
\text { Value }\end{array}$ \\
\hline Sex (\% male) & 42 & 51 & .53 \\
\hline Median age (range/yr) & $69(21-92)$ & 74 (39-92) & .09 \\
\hline Median NIHSS score (range) & $17(6-30)$ & $17(5-32)$ & .68 \\
\hline Median CT ASPECTS (range) & $9(6-10)$ & $9(6-10)$ & .89 \\
\hline CTP (\%) & 44 & 56 & .40 \\
\hline IV rtPA (\%) & 36 & 49 & .29 \\
\hline Vessel occlusion (\%) & & & .88 \\
\hline ICA terminus & 25 & 22 & \\
\hline Cervical ICA + MCA & 4 & 7 & \\
\hline MCA (M1-M2) & 71 & 71 & \\
\hline \multicolumn{4}{|l|}{ Risk factors (\%) } \\
\hline AF & 44 & 53 & .53 \\
\hline HTN & 76 & 80 & .80 \\
\hline DM & 20 & 27 & .62 \\
\hline Smoker & 9 & 9 & .99 \\
\hline HLD & 49 & 49 & .99 \\
\hline Previous stroke/TIA & 7 & 11 & .71 \\
\hline $\begin{array}{l}\text { Median symptom onset-to-groin } \\
\text { puncture time (range/min) }\end{array}$ & $192(78-1193)$ & 219 (27-1097) & .241 \\
\hline $\begin{array}{l}\text { Median symptom onset to reperfusion } \\
\text { time (range/min) }\end{array}$ & $254(107-1245)$ & $279(108-1226)$ & .21 \\
\hline $\begin{array}{l}\text { Median groin puncture to reperfusion } \\
\text { time (range/min) }\end{array}$ & $52(29-225)$ & $61(23-184)$ & .20 \\
\hline Mean No. of passes \pm SD & $2.3 \pm 1.3$ & $2.8 \pm 1.5$ & .09 \\
\hline First-pass reperfusion (\%) & 39 & 23 & .17 \\
\hline Final $\mathrm{mTICI}$ score $(\%)$ & & & $.046^{\mathrm{a}}$ \\
\hline 3 & 51 & 40 & \\
\hline $2 c$ & 16 & 7 & \\
\hline $2 b$ & 31 & 33 & \\
\hline $2 a$ & 2 & 16 & \\
\hline 1 & 0 & 2 & \\
\hline 0 & 0 & 2 & \\
\hline Successful reperfusion $\mathrm{mTICI} \geq 2 \mathrm{~b}(\%)$ & 98 & 80 & $.015^{\mathrm{a}}$ \\
\hline Complete reperfusion $\mathrm{mTICI} 2 \mathrm{c} / 3(\%)$ & 67 & 47 & .09 \\
\hline $\mathrm{mTICl} 3(\%)$ & 51 & 40 & .40 \\
\hline Adjunctive stenting/angioplasty (\%) & 20 & 11 & .26 \\
\hline $\mathrm{SICH}(\%)$ & 2 & 9 & .36 \\
\hline Neurovascular complications (\%) & 4 & 9 & 68 \\
\hline Median mRS at 90 days (range) & $2(0-6)$ & $4(0-6)$ & $.01^{\mathrm{a}}$ \\
\hline $\mathrm{mRS} \leq 2$ at 90 days $(\%)$ & 67 & 43 & $.04^{\mathrm{a}}$ \\
\hline Mortality at 90 days (\%) & 13 & 30 & .10 \\
\hline
\end{tabular}

Note:-AF indicates atrial fibrillation; HTN, hypertension; DM, diabetes mellitus; HLD, hyperlipidemia. ${ }^{a}$ Significant.

treated with the GUARD technique (median, 69 versus 74 years; $P=.09)$, this was not statistically significant.

The GUARD technique demonstrated significantly higher successful TICI $\geq 2$ b reperfusion rates ( $98 \%$ versus $80 \%, P=.015$ ) compared with the standard thrombectomy protocol (Fig $2 A$ ). Additionally, there were trends toward complete TICI $2 c / 3$ reperfusion $(67 \%$ versus $47 \%, P=.09$ ), first-pass recanalization (39\% versus $23 \%, P=$ .17 ), fewer thrombectomy passes (2.3 versus $2.8, P=.09$ ), and faster groin-to-final reperfusion times (52 versus 61 minutes, $P=.2$ ) in the GUARD group, but these did not reach statistical significance (Table 1). Endovascular angioplasty and/or stent placement were required for vessel salvage after revascularization in cases deemed refractory to thrombectomy from underlying vessel wall pathology (atherosclerosis or dissections). There was no difference in the frequency of extracranial carotid angioplasty/stent placement (5 versus 3 interventions) and intracranial stent placement (5 versus 3 interventions) performed in the GUARD ( $9 / 45$ patients) versus standard (5/45 patients) group (20\% versus $11 \%$ respectively, $P=.26$ ).

Safety was assessed with respect to major neurovascular complications, and no difference was noted with GUARD (2/45 patients) versus standard (4/45 patients) thrombectomy techniques (4\% versus $9 \%$ respectively, $P=.68$ ). A non-flowlimiting petrocavernous ICA dissection $(n=1)$ was related to aggressive DGS advancement across the posterior genu segment of the cavernous ICA, which was medically treated with aspirin and without neurologic sequelae. Three suspected iatrogenic, non-flow-limiting intracranial MCA dissections (GUARD $n=1$ versus standard $n=2$ ) were managed medically with IV eptifibatide and postprocedural antiplatelets. Other major iatrogenic complications in the standard thrombectomy group included new territory emboli $(n=2)$ in the fetal posterior cerebral artery distribution and a secondary intraprocedural occlusion of the contralateral ICA. Minor access-site complications included a groin pseudoaneurysm requiring percutaneous thrombin injection in the GUARD group, a carotid puncture-related pseudoaneurysm treated with sonography-guided compression, and 3 minor groin hematomas managed conservatively in the standard group. Furthermore, there were nonsignificant trends toward lower $\mathrm{SICH}$ rates $(2 \%$ versus $9 \%, P=.36)$ and decreased mortality at 90 days (13\% versus $30 \%, P=$ $.10)$ in the GUARD cohort (Table 1).

We found significantly improved clinical outcomes with GUARD thrombectomy, measured by either a median mRS ( 2 versus $4, P=.01)$ or $\mathrm{mRS} \leq 2$ (67\% versus $43 \% ; P=.04)$ at 90 days (Fig $2 B$ ). After adjusting for confounding variables in a multivariable logistic regression model (Table 2), the GUARD technique was independently associated with successful TICI $\geq 2 \mathrm{~b}$ reperfusion (aOR, 13.7; 95\% CI, 1.2-150.9; $P=.03$ ) and good functional $m R S \leq 2$ outcomes $(\mathrm{aOR}=3.77 ; 95 \% \mathrm{CI}, 1.15-12.34$; $P=.03)$. The GUARD technique also demonstrated a strong trend toward complete TICI $2 \mathrm{c} / 3$ reperfusion (aOR, $2.45 ; 95 \% \mathrm{CI}$, $0.93-6.42 ; P=.07)$, but this did not reach statistical significance.

\section{DISCUSSION}

Mechanical thrombectomy via stent retriever and/or largebore DAC aspiration technology has revolutionized the treatment of acute ischemic stroke secondary to ELVO. ${ }^{2-8}$ The success of multiple stroke thrombectomy randomized controlled trials has been attributed in large part to higher reper- 
fusion rates than prior failed intra-arterial thrombolysis/ thrombectomy trials using first- and second-generation devices. ${ }^{19}$ Further work since then has focused on improving stroke processes and thrombectomy techniques to optimize the efficiency and effectiveness of vessel recanalization by reducing times to revascularization, number of required thrombectomy passes, thrombus fragmentation, and distal or new territory embolization.

Large-bore DAC aspiration alone via the ADAPT technique has shown promise as an equivalent alternative to stent retriever-based thrombectomy, ${ }^{3,8,9}$ but several recent studies
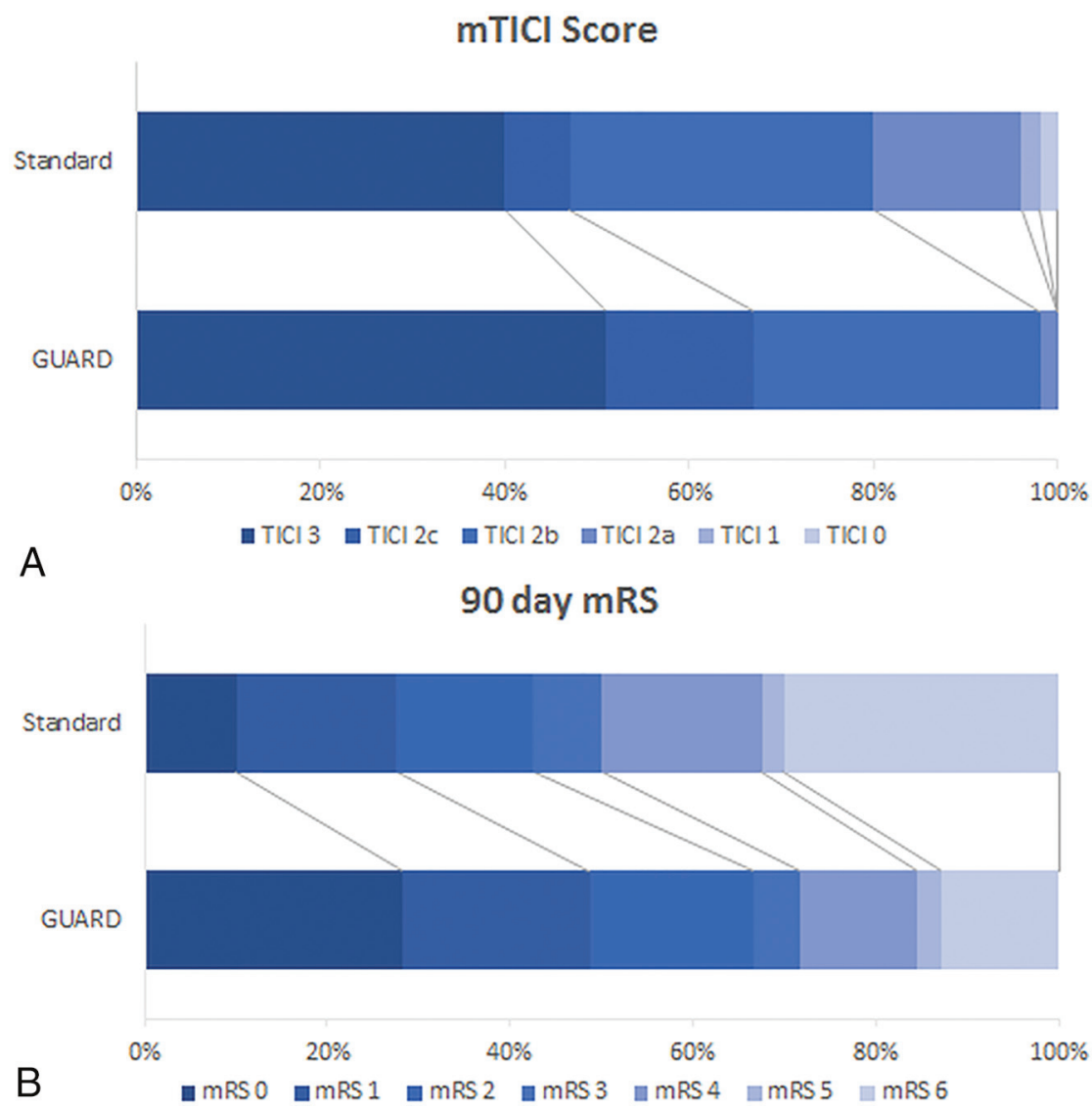

FIG 2. A, Comparison of recanalization results measured with a modified $\mathrm{TICl}$ score between the GUARD and standard techniques $(P=.046)$. $B$, Comparison of functional clinical outcomes by an $\mathrm{mRS}$ at 90 days between the GUARD and standard techniques $(P=.01)$.

Table 2: Multivariable logistic regression analysis with dependent variables for successful reperfusion, complete reperfusion, and independent functional clinical outcomes

\begin{tabular}{|c|c|c|c|c|c|c|}
\hline & \multicolumn{2}{|c|}{ Successful Reperfusion $\mathrm{TICI} \geq 2 \mathrm{~b}$} & \multicolumn{2}{|c|}{ Complete Reperfusion $\mathrm{TICI} 2 \mathrm{c} / 3$} & \multicolumn{2}{|c|}{ Clinical Outcomes mRS $\leq 2$} \\
\hline & aOR & $P$ Value & aOR & $P$ Value & aOR & $P$ Value \\
\hline GUARD & $13.7(1.24-150.85)$ & $.03^{\mathrm{a}}$ & $2.45(0.93-6.42)$ & .07 & $3.77(1.15-12.34)$ & $.03^{\mathrm{a}}$ \\
\hline Age & 1 & .42 & 0.98 & .25 & 1.03 & .19 \\
\hline Sex (male) & 4.2 & .17 & 1.28 & .6 & 2.68 & .09 \\
\hline Onset to groin time & 1.0 & .49 & 1.0 & .18 & 1.0 & .11 \\
\hline IV rtPA & 0.72 & .75 & 0.72 & .54 & 2.71 & .15 \\
\hline \multicolumn{7}{|l|}{ Location } \\
\hline MCA & 9.0 & .14 & 0.78 & .80 & 8.82 & .08 \\
\hline ICA terminus & 4.7 & .35 & 1.06 & .96 & 4.71 & .24 \\
\hline Cervical ICA + MCA & 1 & .286 & 1 & .84 & 1 & .15 \\
\hline CTP & 1.5 & .69 & 1.68 & .32 & 2.82 & .11 \\
\hline
\end{tabular}

Note:-Data reported as adjusted odds ratio (aOR) including 95\% confidence intervals (Cl) for the GUARD technique.

aignificant. 
a 021/027 stent-deployment microcatheter under gentle traction to overcome vascular tortuosity. Furthermore, placement of a $6 \mathrm{~F}$ (8F outer diameter) DGS in 4- to 5-mm diameter ICA segments combined with large-bore DAC aspiration may have sufficiently reduced both antegrade flow and clot-retrieval distance for more effective and efficient thrombectomy.

Failure or incomplete thrombectomy due to distal and new territory embolization has been attributed to limited clot integration during device retrieval across the length and tortuosity of the intracranial vasculature. Combined stent retriever-DAC aspiration techniques increase the retrieval force $\left(\mathrm{F}_{\mathrm{R}}\right)$ and reduce antegrade flow or the impaction force $\left(\mathrm{F}_{\mathrm{I}}\right)$ by using a large-bore (diameter) DAC, but this may be further modified with proximal flow arrest and/or aspiration techniques using a BGC or DGS. While GUARD does not accomplish complete flow arrest akin to a BGC, coaxial DGS placement into the distal petrocavernous segment improves $F_{R}$ vectors in tortuous vasculature and reduces the distance $\left(\right.$ work $=\mathrm{F}_{\mathrm{R}} \times \mathrm{d}$ ) and time $\left(\right.$ momentum $\left.=\mathrm{F}_{\mathrm{R}} \times \mathrm{t}\right)$ required for successful clot retrieval. ${ }^{23} \mathrm{New}$ flexible DGS technology with larger inner diameters (6F 091) is being introduced into the market that will allow safe trackability and access into the distal and smaller ICA segments, but with improved proximal aspiration capacity during retrieval of large-bore DAC-stent retriever complexes to further diminish the $\mathrm{F}_{\mathrm{I}}$.

In our study, we achieved higher rates of successful TICI $\geq 2 \mathrm{~b}$ ( $98 \%$ versus $80 \%$ ) and complete TICI 2 c/3 reperfusion $(67 \%$ versus $47 \%$ ) with an adjunctive GUARD technique compared with propensity score-matched controls using stent retrieverDAC aspiration thrombectomy alone. Indeed with GUARD, we achieved equivalence with the most favorable published results that similarly combined adjunctive BGC aspiration with stent retriever-DAC aspiration thrombectomy (ARTS/ PROTECT), reporting successful and complete reperfusion in $98 \%-100 \%$ and $70 \%$ of patients, respectively. ${ }^{14}$ In contrast, BGC-mediated stent retriever thrombectomy alone without concomitant DAC aspiration has demonstrated relatively lower successful (76\%-79\%) and complete (54\%-58\%) reperfusion rates in the North American Solitaire Stent-Retriever Acute Stroke (NASA) registry and a large BGC meta-analysis. ${ }^{10,11}$ Although statistical trends toward higher single-pass recanalization (39\% versus $23 \%$ ) and shorter procedure times with the GUARD technique did not reach statistical significance, first-pass recanalization was comparable with both the ARTS (43\%) and PROTECT (48\%) techniques. Higher rates of successful and complete reperfusion with GUARD or similarly effective ARTS/PROTECT techniques suggest that adjunctive DGS or BGC devices confer similar benefits. In addition, the SAVE and CAPTIVE techniques using proximal aspiration from a cervical guide sheath and/or continuous DAC aspiration during stent retriever deployment instead of a BGC also yielded excellent complete TICI $2 \mathrm{c} / 3(78 \%-80 \%)$ and firstpass (59\%-72\%) reperfusion rates, superior to stent retrieverDAC aspiration alone techniques (SOLUMBRA). ${ }^{5-7}$ Interestingly, most cases using SAVE used $8 \mathrm{~F}$ guide catheters with diameters equivalent to those in the 6F 088 DGS used in our GUARD technique.

Studies yielding the highest reperfusion rates combined stent retriever thrombectomy with both DAC and proximal BGC/DGS aspiration and resulted in the best clinical outcomes (ARTS, SAVE, PROTECT, GUARD). Our improved reperfusion rates in the GUARD cohort correlated with significantly higher rates of functional independence (67\% versus $43 \%$, mRS $\leq 2$ at 90 days, $P=.01$ ), equivalent to previously published studies using BGCs and/or proximal aspiration in either the large BGC meta-analysis $(60 \%)^{10}$ or the single-arm SAVE (59\%) and ARTS (66\%) studies. Although the PROTECT technique failed to report 90-day clinical outcomes, prior description of this combined stent retriever with DAC and proximal BGC aspiration approach by Stampfl et $\mathrm{al}^{13}$ noted 52\% mRS $0-2$ at 90 days. ${ }^{13,14}$ Further larger cohort comparative studies of our GUARD technique using DGS aspiration against similarly effective and adjunctive BGC aspiration techniques are warranted and planned in a multicenter setting.

Several limitations in our study include a retrospective, nonrandomized study design. We attempted to mitigate this risk with propensity score matching to provide case controls without statistically significant differences in baseline parameters. In addition, a multivariate logistic regression analysis was performed to investigate the independent effect of the GUARD technique on reperfusion and good functional outcomes, while adjusting for possible confounders. However, despite propensity score matching, a nonstatistical trend toward a younger age bias in the GUARD group persisted. Our study may have been prone to patient-selection bias as DGS advancement could have been hindered in challenging aortic arch anatomy or $360^{\circ}$ cervical vascular loops. We identified 7 cases in which we failed to advance a DGS into the petrocavernous ICA segments to initiate the GUARD technique, and 4 of these cases had a $360^{\circ}$ loop in the proximal ICA. Despite traversing the vascular loops with the DGS, inadequate catheter length prevented further distal access into the petrocavernous ICA, which may have been mitigated if longer (100 $\mathrm{cm}$ ) DGSs were used or available. The remaining 3 cases presented with extremely tortuous aortic and/or great vessel anatomy preventing either distal DGS placement $(n=1)$ or requiring direct carotid access $(n=2)$, with short introducer sheaths limited to the cervical ICA. However, there were no cases in which the GUARD technique was possible and then abandoned for another technique to achieve successful recanalization. In contrast, we reverted to the GUARD technique from our standard technique in 3 patients after several failed thrombectomy attempts; these patients were analyzed within the GUARD cohort, potentially negatively impacting procedure times, number of passes, and percentage of first-pass recanalizations.

Finally, caution is warranted in comparing studies on adjunctive thrombectomy techniques, especially when combining stent retrieval, DAC aspiration, and/or proximal BGC/DGS aspiration. It may be difficult to control for the various types and sizes of thrombectomy devices or other uncontrolled techniques such as positioning or deployment of devices, time for thrombus integration/engagement, and retrieval techniques. Furthermore, most studies to date have been nonrandomized, single-arm, and smallsample-size studies without control populations. Nevertheless, the only comparative studies with internal control groups remain CAPTIVE, PROTECT, and the current GUARD study, all dem- 
onstrating relatively higher reperfusion rates and better clinical outcomes.

\section{CONCLUSIONS}

We describe a novel GUARD technique using a flexible 6F 088 DGS that is advanced into the petrocavernous segment of the ICA during combined stent retriever-DAC aspiration thrombectomy. In comparison with a propensity score-matched patient cohort, the adjunctive GUARD technique was shown to be safe and independently effective in improving both reperfusion and functional clinical outcomes. Our results using GUARD appear equivalent to the most optimum techniques published to date, including proximal BGC aspiration in combination with stent retriever-DAC aspiration thrombectomy.

\section{REFERENCES}

1. Powers WJ, Rabinstein AA, Ackerson T, et al; American Heart Association Stroke Council. 2018 Guidelines for the Early Management of Patients with Acute Ischemic Stroke: a Guideline for Healthcare Professionals from the American Heart Association/American Stroke Association. Stroke 2018;49:e46-110 CrossRef Medline

2. Goyal M, Menon BK, van Zwam WH, et al; HERMES collaborators. Endovascular thrombectomy after large-vessel ischaemic stroke: a meta-analysis of individual patient data from five randomised trials. Lancet 2016;387:1723-31 CrossRef Medline

3. Turk AS, Frei D, Fiorella D, et al. ADAPT FAST study: a direct aspiration first pass technique for acute stroke thrombectomy. $J \mathrm{Neu}$ rointerv Surg 2014;6:260-64 CrossRef Medline

4. Lee HC, Kang DH, Hwang YH, et al. Forced arterial suction thrombectomy using distal access catheter in acute ischemic stroke. $\mathrm{Neu}$ rointervention 2017;12:45-49 CrossRef Medline

5. Humphries W, Hoit D, Doss VT, et al. Distal aspiration with retrievable stent assisted thrombectomy for the treatment of acute ischemic stroke. J Neurointerv Surg 2015;7:90-94 CrossRef Medline

6. McTaggart RA, Tung EL, Yaghi S, et al. Continuous aspiration prior to intracranial vascular embolectomy (CAPTIVE): a technique which improves outcomes. J Neurointerv Surg 2017;9:1154-59 CrossRef Medline

7. Maus V, Behme D, Kabbasch C, et al. Maximizing first-pass complete reperfusion with SAVE. Clin Neuroradiol 2018;28:327-33 CrossRef Medline

8. Lapergue B, Blanc R, Gory B, et al; ASTER Trial Investigators. Effect of endovascular contact aspiration vs stent retriever on revascularization in patients with acute ischemic stroke and large vessel occlusion: the ASTER Randomized Clinical Trial. JAMA 2017;318: 443-52 CrossRef Medline

9. Turk AS 3rd, Siddiqui A, Fifi JT, et al. Aspiration thrombectomy versus stent retriever thrombectomy as first-line approach for large vessel occlusion (COMPASS): a multicentre, randomised, open label, blinded outcome, non-inferiority trial. Lancet 2019;393:9981008 CrossRef Medline
10. Brinjikji W, Starke RM, Murad MH, et al. Impact of balloon guide catheter on technical and clinical outcomes: a systematic review and metaanalysis. J Neurointerv Surg 2018;10:335-39 CrossRef Medline

11. Nguyen TN, Malisch T, Castonguay AC, et al. Balloon guide catheter improves revascularization and clinical outcomes with the Solitaire device: analysis of the North American Solitaire Acute Stroke Registry. Stroke 2014;45:141-45 CrossRef Medline

12. Massari F, Henninger N, Lozano JD, et al. ARTS (Aspiration-Retriever Technique for Stroke): initial clinical experience. Interv Neuroradiol 2016;22:325-32 CrossRef Medline

13. Stampfl S, Pfaff J, Herweh C, et al. Combined proximal balloon occlusion and distal aspiration: a new approach to prevent distal embolization during neurothrombectomy. J Neurointerv Surg 2017;9: 346-51 CrossRef Medline

14. Maegerlein C, Monch S, Boeckh-Behrens T, et al. PROTECT: PRoximal balloon Occlusion TogEther with direCt Thrombus aspiration during stent retriever thrombectomy: evaluation of a double embolic protection approach in endovascular stroke treatment. J Neurointerv Surg 2018;10:751-55 CrossRef Medline

15. Albers GW, Marks MP, Kemp S, et al; DEFUSE 3 Investigators. Thrombectomy for stroke at $\mathbf{6}$ to $\mathbf{1 6}$ hours with selection by perfusion imaging. N Engl J Med 2018;378:708-18 CrossRef Medline

16. Nogueira RG, Jadhav AP, Haussen DC, et al; DAWN Trial Investigators. Thrombectomy $\mathbf{6}$ to $\mathbf{2 4}$ hours after stroke with a mismatch between deficit and infarct. $N$ Engl J Med 2018;378:11-21 CrossRef Medline

17. Hacke W, Kaste M, Bluhmki E, et al. Thrombolysis with alteplase 3 to 4.5 hours after acute ischemic stroke. $N$ Engl J Med 2008;359: 1317-29 CrossRef Medline

18. Goyal M, Fargen KM, Turk AS, et al. 2C or not $2 \mathrm{C}$ : defining an improved revascularization grading scale and the need for standardization of angiography outcomes in stroke trials. J Neurointerv Surg 2014;6:83-86 CrossRef Medline

19. Broderick JP, Palesch YY, Demchuk AM, et al; Interventional Management of Stroke (IMS) III Investigators. Endovascular therapy after intravenous t-PA versus t-PA alone for stroke. $N$ Engl J Med 2013;368:893-903 CrossRef Medline

20. Shah VA, Martin CO, Hawkins AM, et al. Groin complications in endovascular mechanical thrombectomy for acute ischemic stroke: a 10-year single center experience. J Neurointerv Surg 2016;8:568-70 CrossRef Medline

21. Frank JJ, Kamalakannan D, Kodenchery M, et al. Retroperitoneal hematoma in patients undergoing cardiac catheterization. J Interv Cardiol 2010;23:569-74 CrossRef Medline

22. Trimarchi S, Smith DE, Share D, et al; BMC2 Registry. Retroperitoneal hematoma after percutaneous coronary intervention: prevalence, risk factors, management, outcomes, and predictors of mortalitya report from the BMC2 (Blue Cross Blue Shield of Michigan Cardiovascular Consortium) registry. JACC Cardiovasc Interv 2010;3:845-50 CrossRef Medline

23. Yoo AJ, Andersson T. Thrombectomy in acute ischemic stroke: challenges to procedural success. J Stroke 2017;19:121-30 CrossRef Medline 